\title{
A sub-micron plasmonic nano-resonator refractometer in metal-coated fiber taper
}

\author{
Ming Ding*a, ${ }^{\mathrm{a} b}$, Michalis N. Zervas ${ }^{\mathrm{b}}$, Wei Quan ${ }^{\mathrm{a}}$, Gilberto Brambilla ${ }^{\mathrm{b}}$ \\ ${ }^{a}$ School of Instrument Science and Opto-Electronics Engineering, Beihang University, Beijing, \\ China 100191; \\ ${ }^{\mathrm{b}}$ Optoelectronics Research Centre, University of Southampton, Highfield, Southampton, UK \\ SO171BJ.
}

\begin{abstract}
Plasmonic slot nano-resonators (PSNR) embedded in gold-coated optical fiber tapers, can show strong three dimensional localization when optimized. Here the PSNR is used as a refractometer with small footprint. An average sensitivity of $66.7 \mathrm{~nm} / \mathrm{RIU}$ and a resolvable index change of $1.5 \times 10^{-4}$ for a resolvable wavelength change of $0.01 \mathrm{~nm}$ are obtained.
\end{abstract}

Keywords: plasmonic nano-resonator, enhancement factor, focused ion beam, refractive index sensor

\section{INTRODUCTION}

In recent years, research in plasmonics has attracted considerable interest because of their extraordinary potentials in energy confinement and nanoantennas. Nanoscale apertures in thin noble-metal films, with dimensions comparable to the light wavelength, can form plasmonic nano-resonators and show astonishing optical properties leading to enhanced and selective light transmission and confinement. A number of different nano-structures have been considered and studied in detail for their ability to concentrate light. These include circular, square, rectangular, bow-tie, as well as, L-, C- and $\mathrm{H}-$ shaped nano-structures [1]. At resonance, such structures can concentrate an incident light field into a small volume with orders-of-magnitude intensity enhancement. In contrast to conventional optical systems using lenses and mirrors, plasmonics nano-resonators can provide hot spots in the near field with dimensions determined mainly by the structure size rather than by the diffraction limit. So far, the transmission properties of slot nano-resonators have been studied under plane-wave excitation directed perpendicularly to the plane of the resonator [2, 3]. In this paper, we study theoretically a strongly-coupled 3D plasmonic slot nano-resonator (PSNR) by embedding a slot nano-cavity in a plasmonic cylindrical waveguide formed by a thin-metal-film coated fiber taper. Its refractive index response is simulated. Also, for the first time, both theoretically and experimentally, the transverse excitation of a strongly-coupled 3D PSNR is investigated by embedding a rectangular slot nano-cavity in a plasmonic structure formed by a thin-metalfilm coated optical fiber tip.

\section{PLASMONIC SLOT NANO-RESONATOR SIMULATION}

Theoretical analysis was carried out using 3D Finite Element Method (FEM) to numerically solve Maxwell equations in the frequency domain for the proposed designs. The numerical model was defined, solved and analyzed using the commercial software COMSOL 4.1 Multiphysics. Figure 1 shows the structure used for the modeling. A plasmonic slot nano-cavity was embedded on the side of $30 \mathrm{~nm}$ gold-coated fiber taper (see Figure 1 (a)). Figure 1 (b) gives the topview of the two PSNRs considered in this study, namely a bow-tie nano-cavity with $L$ length, $D$ width and $d$ waist width and a rectangular slot nano-cavity with dimensions $D$ and $L$. The depth of the slot nano-cavities is equal to the thickness $(t)$ of gold layer. In all calculations $t=30 \mathrm{~nm}$. Light is launched from one of the fiber pigtails into the $1 \mu \mathrm{m}$ diameter taper and the various resonances can be identified simply monitoring the transmitted and reflected spectral features.

Figure 2 (a) shows the transmissivity and the reflectivity of the composite structure when the bow-tie PSNR has $L=400 \mathrm{~nm}, D=200 \mathrm{~nm}$, and $d=34.4 \mathrm{~nm}$. There are several dips observed in the transmission spectrum and associated peaks in the reflection spectrum, which corresponds to different type of resonances of the embedded PSNR. In such a 
strongly coupled system where the embedded cavity is integral part of the plasmonic waveguide, resonances are associated with impedance mismatch of the propagating waves and multiple reflections at the nano-resonator edges (effective Fabry-Perot cavity). Three main resonances were identified: the one at $\lambda=880 \mathrm{~nm}$ corresponds to a secondorder resonance, with two intensity maxima along the length of the bow-tie PSNR. The resonances at $\lambda=1524 \mathrm{~nm}$ and $2050 \mathrm{~nm}$ correspond to first-order PSNR resonances, with only one intensity maximum along the bow-tie length.
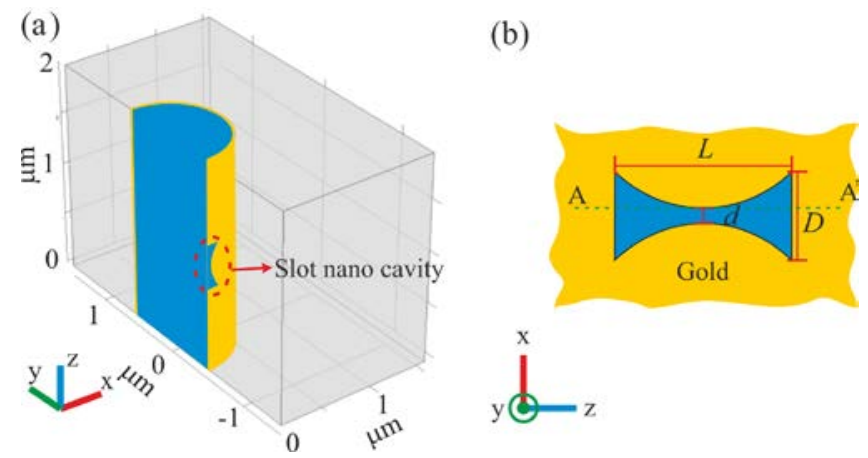

Figure 1 (a) Geometry of the PSNR embedded in the plasmonic microfiber; (b) top-view of the bow-tie.

Figure 2 (b) shows the total electric field modulus $(|E|)$ at several wavelengths corresponding to the major $(\lambda=880$ $\mathrm{nm}, 1524 \mathrm{~nm}$ and $2050 \mathrm{~nm})$ resonances, as well as some minor $(\lambda=820 \mathrm{~nm}, 970 \mathrm{~nm}, 1040 \mathrm{~nm}, 1170 \mathrm{~nm}$ and $1260 \mathrm{~nm})$ resonances. The intensity is plotted on a plane tangential to the top resonator surface. At the major resonances, the electric field is highly localized and centered at the bow-tie waist. At the minor resonances, the corresponding electric field distributions are gradually shifting off-center and their peak values decrease. A single resonator shows enhancement factor in excess of $9 \times 10^{5}$, which we believe is the biggest enhancement factor calculated in all types of nano-resonators so far.

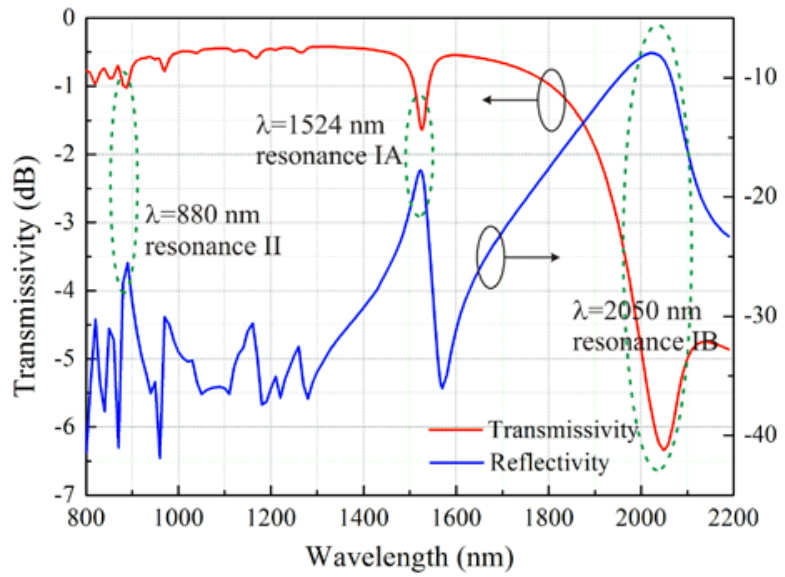

(a)

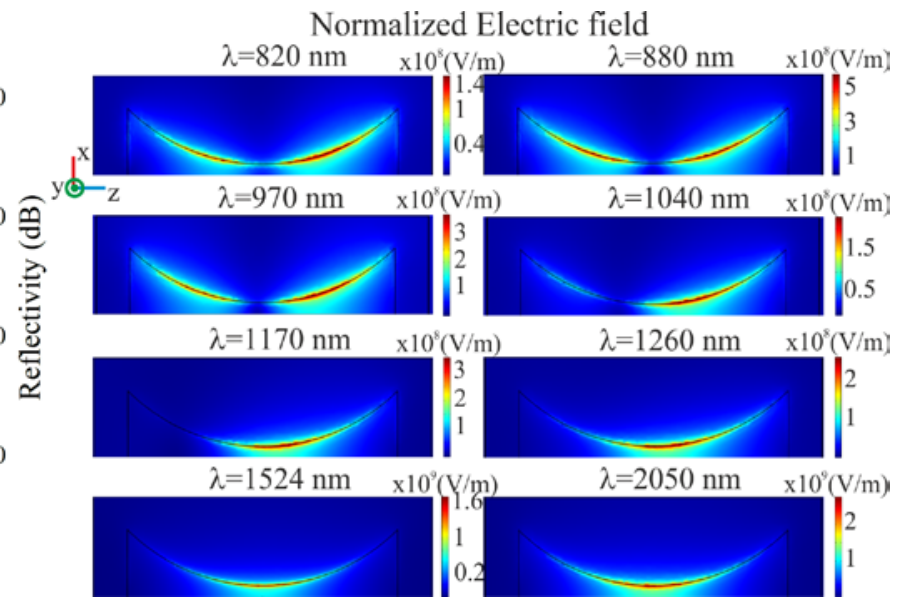

(b)

Figure 2 Transmissivity (red) and reflectivity (blue) of the bow-tie PSNR embedded in the plasmonics; (b) the normalized electric field on $\mathrm{x}-\mathrm{z}$ plane for bow-tie PSNR at different wavelengths $\lambda$.

Different bow-tie PSNRs with different waist and different edge width, multiple cascaded bow-tie PSNR, and rectangular PSNR were numerically investigated [4]. Wavelength shift rates were found to be strongly dependent on the nature of the associated resonance and the plasmonic waveguide characteristics.

Since the PSNR has extremely small size (less than $0.1 \mu^{2}$ ) and it is sensitive to the surrounding environment, the PSNR can be used as a compact refractometer in small areas. The refractive index (RI) dependence of PSNR is analyzed by changing the surrounding RI. 
Figure 3 (a) presents the transmissivity of PSNR when the surrounding RI changes from $n=1$ to $n=1.15$ in steps of 0.05. For increasing RIs, the peak at $1524 \mathrm{~nm}$ monotonically shifts to longer wavelengths. The relationship between the wavelength of the peak and the refractive index of surrounding is shown in Figure 3 (b). The wavelength changes linearly with the refractive index. The PSNR sensitivity, defined as the ratio between the wavelength shift and the RI change, resulted to be $66.7 \mathrm{~nm} / \mathrm{RIU}$, with a resolvable index change of $1.5 \times 10^{-4}$ for a resolvable wavelength change of $0.01 \mathrm{~nm}$

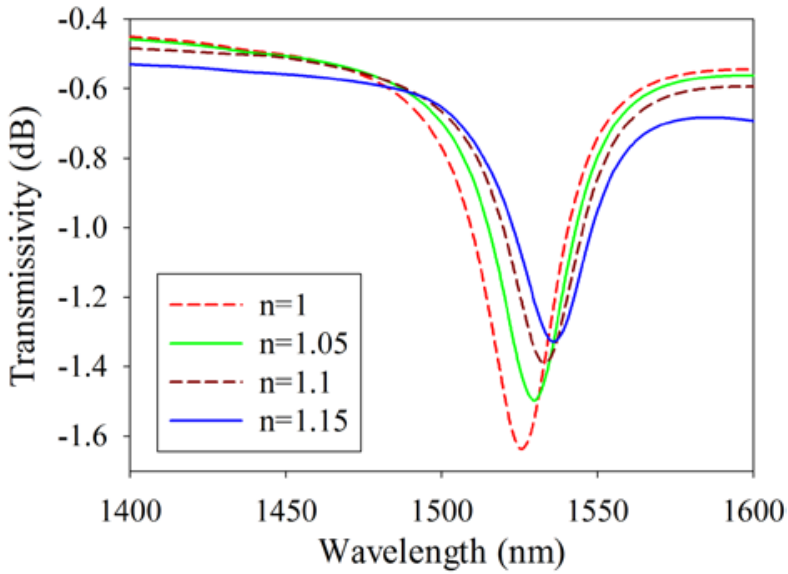

(a)

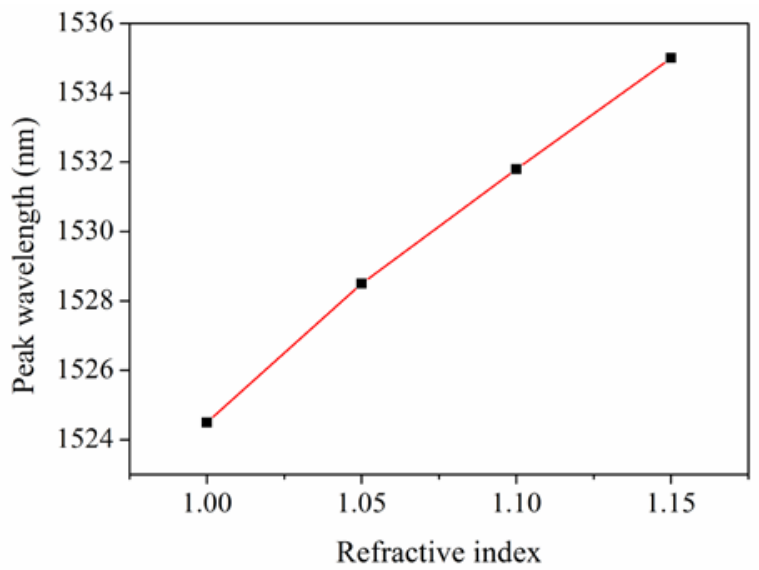

(b)

Figure 3 (a) The transmissivity of PSNR when the surrounding RI changes from $n=1$ to $n=1.15$; (b) The relationship between the wavelength of the peak and the refractive index of surrounding.

\section{PLASMONIC SLOT NANO-RESONATOR EXPERIMENT}

A rectangular PSNR was inscribed on the surface of a gold-coated fiber tip. The fabrication process involved three main steps: manufacture of optical fiber tip, deposition of a thin gold layer and focused ion beam (FIB) nanopatterning [5].

The optical fiber tips were manufactured using a commercial micropipette puller (P-2000) (Sutter Instrument Inc., Novato, USA) which is a $\mathrm{CO}_{2}$ laser based pulling machine controlled by a microprocessor. Since high transmission efficiency is targeted in this paper, the taper angle of the optical fiber tips has to be small enough to adiabatically convert the fundamental mode in the fiber core into a fundamental mode in the microfiber [6]. $\mathrm{CO}_{2}$ laser power, pulling speed, tension and delays were optimized to maximize the adiabaticity of the transition region between the optical fiber and fiber tip. A $30 \mathrm{~nm}$ thickness layer of gold was then deposited on the tips using an E-beam evaporator to induce the plasmonic metal layer and to avoid charging during FIB processing; indeed, charge accumulation is a major issue and seriously degrades FIB imaging and processing capabilities. The gold-coated plasmonic fiber tips were then nanostructured using a FIB milling system. As FIB is a direct writing technique with resolution smaller than $30 \mathrm{~nm}$, it offers extreme flexibility and allows for high precision in the nanostructuring. By FIB, the apex of the plasmonic fiber tips was flat cut at the diameter $\sim 1 \mu \mathrm{m}$ and a rectangular gold layer with $400 \mathrm{~nm}$ length and $200 \mathrm{~nm}$ width was milled out to form a PSNR at the position when the diameter is $\sim 1.22 \mu \mathrm{m}$.

The SEM image of the rectangular PSNR on a plasmonic fiber tip and its magnification image are shown in Figure 4 (a) and (b). However, the gold layer was slightly damaged by the re-deposition of silica during milling. The milling impact on surface quality could be improved by using ion beam with small currents. The optical properties of the PSNR embedded in plasmonic fiber tips were characterized with the set-up shown in Figure 4 (c). Many resonance features were observed in the reflectivity of the samples recorded at the polarization perpendicular to the PSNR (Figure 4 (d) red solid curve). The simulations (blue dashed curve in Figure 4 (d)) were modeled with the dimensions obtained from the SEM image. The biggest enhancement factor $7.24 \times 10^{3}$ is observed numerically at resonance $\lambda=1450 \mathrm{~nm}$, which is few times larger than that at the other resonance wavelengths. The two curves in Figure 4 (d) show a number of differences, like different resonance peak positions, slightly different reflection amplitudes and number of peaks. A possible explanation might be related to imperfect taper and gold layer surfaces and to particles attached to the gold layer surface. Particles induce plasmonic effects even when their size is small. Moreover, part of the gold layer was removed due to the 
re-deposition of material in the FIB processing and formed additional extremely small nano-cavities. This not only causes plasmonic cavities but also changes the interference condition in the plasmonic fiber tip.

(a)

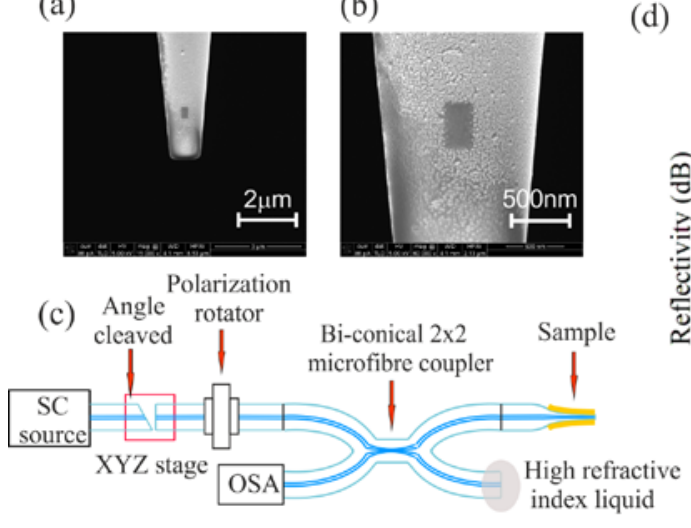

(d)

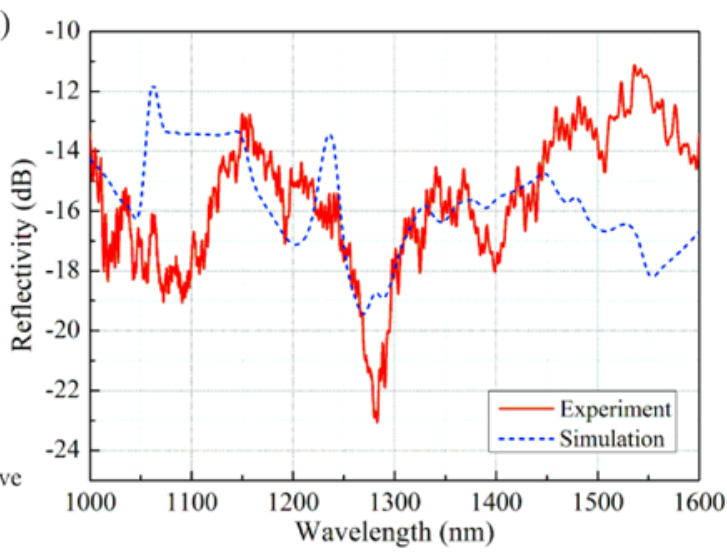

Figure 4 (a) SEM image of the plasmonic fiber tip with a rectangular PSNR; (b) magnified image of the cavity area; (c) Schematic of experimental set-up to characterize the PSNR spectral properties; (d) reflectivity spectrum of the sample in the experiment (the red solid curve) and in the simulation (the blue dashed curve).

\section{CONCLUSION}

In this paper, a PSNR embedded in thin-gold metal film coated plasmonic fiber taper, was numerically and experimentally investigated. A strong three dimensional localization is observed in simulations. The wavelength shift when the surrounding RI changes is analyzed, resulting in an average sensitivity of $66.7 \mathrm{~nm} / \mathrm{RIU}$ and a resolvable index change of $1.5 \times 10^{-4}$ for a resolvable wavelength change of $0.01 \mathrm{~nm}$. A rectangular PSNR embedded in a thin gold-coated plasmonic fiber tip was experimentally demonstrated. The strongest enhancement factor $7.24 \times 10^{3}$ was achieved for the resonance at the wavelength $\lambda \sim 1450 \mathrm{~nm}$, recorded when light was polarized perpendicularly to the PSNR. FIB milling was used in the experiments to inscribe a PSNR on the gold-coated plasmonic fiber tip. The refractive index dependence of PSNR experiment is in progress. The PSNR nano-scale dimensions promise the development of refractometric sensors capable of measuring minute nano-litre quantities.

\section{ACKNOWLEDGMENTS}

GB gratefully acknowledges the Royal Society (London, UK) for his University Research Fellowship.

\section{REFERENCES}

[1] Lee, B., Lee, I. M., Kim, S., Oh, D. H., and Hesselink, L., "Review on subwavelength confinement of light with plasmonics," J Mod Optic , 57, 1479-1497 (2010)

[2] Sederberg, S., and Elezzabi, A. Y., "Nanoscale plasmonic contour bowtie antenna operating in the mid infrared," Opt Express, 19, 15532-15537 (2011)

[3] Jin, E. X. and Xu, X. F., "Enhanced optical near field from a bowtie aperture," Appl. Phys. Lett., 88, 153110 (2006)

[4] M. Ding, G. Brambilla, M. N. Zervas, "Plasmonic slot nano-resonators embedded in metal-coated plasmonic microfibers,” J. Lightwave. Technol. 31, 3093-3103 (2013)

[5] M. Ding, M. N. Zervas, and G. Brambilla, "Transverse excitation of plasmonic slot nano-resonators embedded in metal-coated microfiber tips,” Appl. Phys. Lett. 102, 141110 (2013)

[6] Love, J. D., "Spot size, adiabaticity and diffraction in tapered fibres,” Electron. Lett. 23, 993-994 (1987) 